\title{
Transmission Electron Microscopy on Interface Engineered Superconducting Thin Films
}

\author{
Sara Bals, Gustaaf Van Tendeloo, Guus Rijnders, Mark Huijben, Victor Leca, and Dave H. A. Blank
}

\begin{abstract}
Transmission electron microscopy is used to evaluate different deposition techniques, which optimize the microstructure and physical properties of superconducting thin films. High-resolution electron microscopy proves that the use of an $\mathrm{YBa}_{2} \mathrm{Cu}_{2} \mathrm{O}_{x}$ buffer layer can avoid a variable interface configuration in $\mathrm{YBa}_{2} \mathrm{Cu}_{3} \mathrm{O}_{7-\delta}$ thin films grown on $\mathrm{SrTiO}_{3}$. The growth can also be controlled at an atomic level by using sub-unit cell layer epitaxy, which results in films with high quality and few structural defects.

Epitaxial strain in $\mathrm{Sr}_{0.85} \mathrm{La}_{0.15} \mathrm{CuO}_{2}$ infinite layer thin films influences the critical temperature of these films, as well as the microstructure. Compressive stress is released by a modulated or a twinned microstructure, which eliminates superconductivity. On the other hand, also tensile strain seems to lower the critical temperature of the infinite layer.
\end{abstract}

Index Terms-High resolution electron microscopy, interface structure, superconducting thin films.

\section{INTRODUCTION}

$\mathbf{O}$ $\mathrm{NE}$ of today's challenges when growing high- $T_{c}$ superconducting thin films is to obtain films with optimal physical properties and atomically flat surfaces. The latter requirement is of crucial importance for the application of the films in planar multilayer devices. Surface morphology of thin films is strongly depending on conditions such as deposition parameters, substrate quality, etc. To optimize these conditions, a thorough understanding of thin film growth is indispensable. Particularly, the initial growth of the films, including the interface structure with the substrate is of extreme importance and must therefore be controlled and characterized on atomic level.

Controlling and manipulating interfaces is possible using advanced deposition techniques such as sub-unit cell layer epitaxy [1], a technique in which sub-unit cell layers are sequentially deposited by pulsed layer deposition (PLD) until the desired compound is formed. Film growth can be monitored in situ by reflection high-energy electron diffraction (RHEED) [2] and after growth the surface morphology can be characterized using atomic force microscopy (AFM).

Manuscript received August 5, 2002. This work has been performed with financial support of IUAP V-1 and the Fund for Scientific Research-Flanders (FWO).

S. Bals is with EMAT, University of Antwerp (RUCA), B-2020 Antwerp, Belgium (e-mail: sabals@ruca.ua.ac.be).

G. Van Tendeloo is with EMAT, University of Antwerp (RUCA), B-2020 Antwerp, Belgium (e-mail: gvt@ ruca.ua.ac.be).

G. Rijnders, M. Huijben, V. Leca, and D.H.A. Blank are with the Low Temperature Division, Applied Physics and MESA + Research Institute, Applied Physics, University of Twente, 7500 AE Enschede, The Netherlands (e-mail: a.j.h.m.rijnders@tn.utwente.nl; m.huijben@tn.utwente.nl; v.leca@tn.utwente.nl; d.h.a.blank@tn.utwente.nl).

Digital Object Identifier 10.1109/TASC.2003.812023
However, to characterize initial film growth on an atomic scale, transmission electron microscopy (TEM) and particularly high-resolution electron microscopy (HREM) is required.

Using HREM, a variable interface configuration was found in ultra-thin $\mathrm{DyBa}_{2} \mathrm{Cu}_{3} \mathrm{O}_{7-\delta}$ (Dy123) films grown on single $\mathrm{TiO}_{2}$ terminated $\mathrm{SrTiO}_{3}$ (STO) substrates [3]. This results in antiphase boundaries (APB's) and very rough film surfaces already during initial deposition. In this study, TEM shows that a variable interface configuration in $\mathrm{YBa}_{2} \mathrm{Cu}_{3} \mathrm{O}_{7-\delta}(\mathrm{Y} 123)$ thin films can be avoided by using a single $\mathrm{YBa}_{2} \mathrm{Cu}_{2} \mathrm{O}_{x}$ (Y122) unit cell layer as a buffer layer or by using sub-unit cell layer epitaxy.

Physical properties of thin films are also influenced by the substrate and deposition parameters, which is demonstrated here for infinite layer (IL) thin films. Although these compounds have a very simple structure, it is apparently not evident to obtain thin films with good physical properties. Epitaxial strain will influence microstructural properties and therefore the superconducting properties. It can even completely eliminate superconductivity. The choice of the correct substrate is therefore essential [4].

HREM was used to study the microstructure of $\mathrm{Sr}_{0.85} \mathrm{La}_{0.15} \mathrm{CuO}_{2}$ thin films deposited on different substrates, with respect to their critical temperature.

\section{EXPERIMENTAL}

\section{A. Thin Film Growth}

Dy123, Y123 and $\mathrm{Sr}_{0.85} \mathrm{La}_{0.15} \mathrm{CuO}_{2}$ films were deposited using PLD $(\lambda=248 \mathrm{~nm})$, whereas for Y122 and the sub-unit cell layer epitaxy, pulsed laser interval deposition (PLiD) [5] was used. The growth was monitored in situ by RHEED and ex situ by AFM and X-ray diffraction (XRD). STO and $\mathrm{NdGaO}_{3}$ (NGO) substrates are treated as described by [6], resulting in atomically smooth $\mathrm{TiO}_{2}$ and $\mathrm{GaO}_{x}$ terminated substrates, respectively. $\mathrm{KTaO}_{3}$ (KTO) substrates are used as received.

1) Y123 Thin Films: Initial growth of Y123 films on STO is manipulated by first depositing a single unit cell layer from a target with cation ratio $\mathrm{Y}: \mathrm{Ba}: \mathrm{Cu}$ equal to $1: 2: 2$ before subsequent deposition of Y123.

Also sub-unit cell layer epitaxy is applied for growth of Y123 by sequential deposition of two $\mathrm{BaCuO}_{x}$ unit cell layers and one $\mathrm{YCuO}_{x}$ unit cell layer [1] on NGO.

2) IL Thin Films: Films with a thickness of approximately $130 \mathrm{~nm}$ were deposited on STO and KTO, both with and without $\mathrm{BaTiO}_{3}$ (BTO) buffer layer. Films on STO were annealed for $1 \mathrm{~h}$ at $450{ }^{\circ} \mathrm{C}$ in an $\mathrm{O}_{2} / \mathrm{N}_{2}$ environment or cooled down in an $\mathrm{N}_{2}$ environment. 


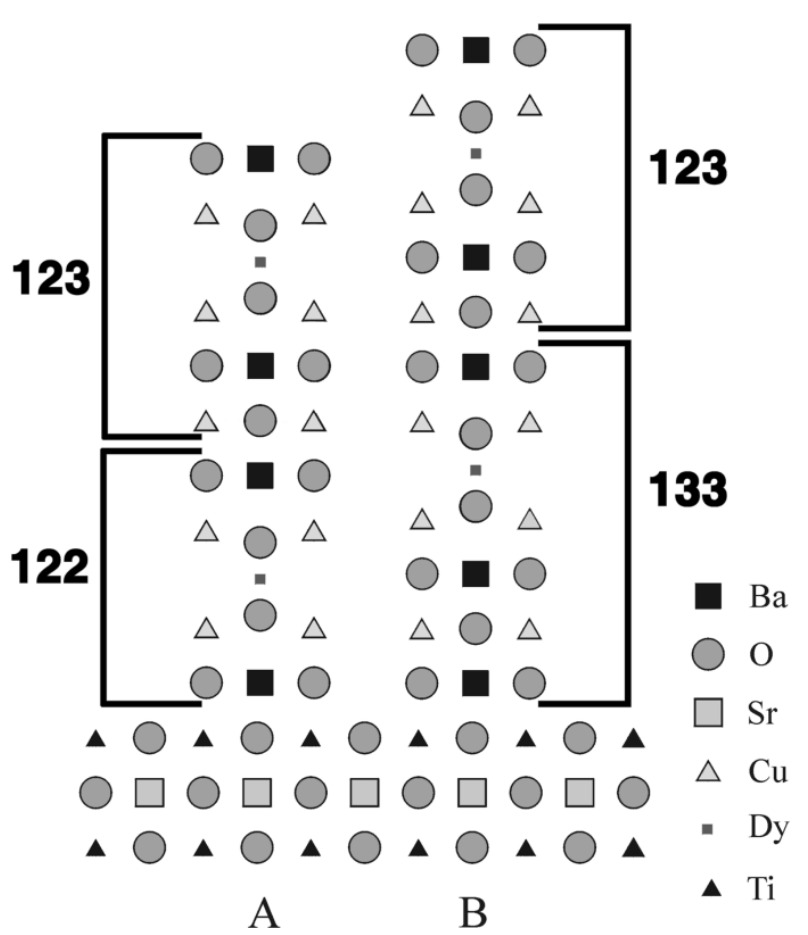

Fig. 1. Different interface stackings in Dy123 films on $\mathrm{TiO}_{2}$ terminated STO: (a) bulk-Bao- $\mathrm{CuO}_{2}-\mathrm{RE}-\mathrm{CuO}_{2}-\mathrm{BaO}-\mathrm{CuO}-$ bulk; (b) bulk-BaO-CuO-BaO$\mathrm{CuO}_{2}$ - $\mathrm{RE}-\mathrm{CuO}_{2}$-bulk.

\section{B. Electron Microscopy}

Films grown on STO and KTO were cut parallel to the (010) plane of STO/KTO, whereas films grown on NGO were cut parallel to the (110) plane of NGO, which means parallel to the (010) or (100) plane of the Y123 film, since the $a$ and $b$-directions of the film are rotated in plane over $45^{\circ}$ with respect to those of the substrate. After mechanically polishing the sample down to a thickness of $10 \mu \mathrm{m}$, electron transparency was obtained by ion milling. HREM was done with a JEOL 4000EX, having a Scherzer resolution of $0.17 \mathrm{~nm}$.

\section{RESULTS AND DISCUSSION}

\section{A. Manipulation of RE123 Thin Film}

Previous studies of ultra-thin Dy123 films grown on single $\mathrm{TiO}_{2}$ terminated STO, showed the presence of a variable atomic stacking at the interface [3]. Two interface configurations (presented in Fig. 1) apparently have the same formation probability. As a consequence, APB's are formed during initial growth when islands with a different interface stacking coalescence. Furthermore, already during initial growth, very rough film surfaces are formed [1], [3]. The APB's will influence the superconducting properties of the film, since one out of two superconducting $\mathrm{CuO}_{2}$ planes will be disconnected. Growing thicker films, APB's may end in dislocations, leading to the formation of growth spirals at the surface of the film [7].

Obviously, a variable interface configuration has to be avoided and therefore, two deposition methods are used to obtain films with a single type of interface configuration:

1) Using an Y122 Buffer Layer: As can be seen in Fig. 1 the first unit cell of the Y123 film in model A is a is an Y122 unit cell rather than an Y123 unit cell. The film growth can

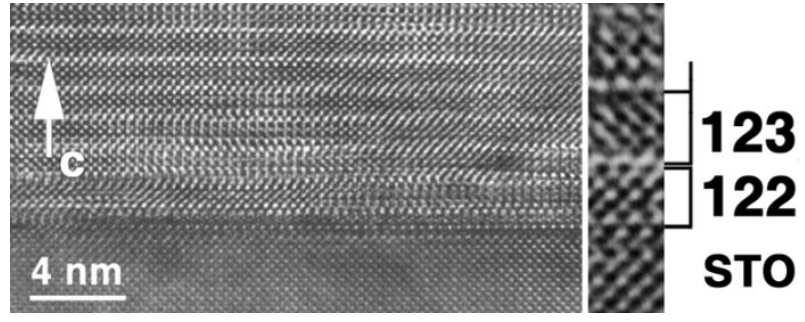

Fig. 2. HREM image of an Y123 film with a single unit cell of Y122 as buffer layer. An enlargement of the interface is also shown.

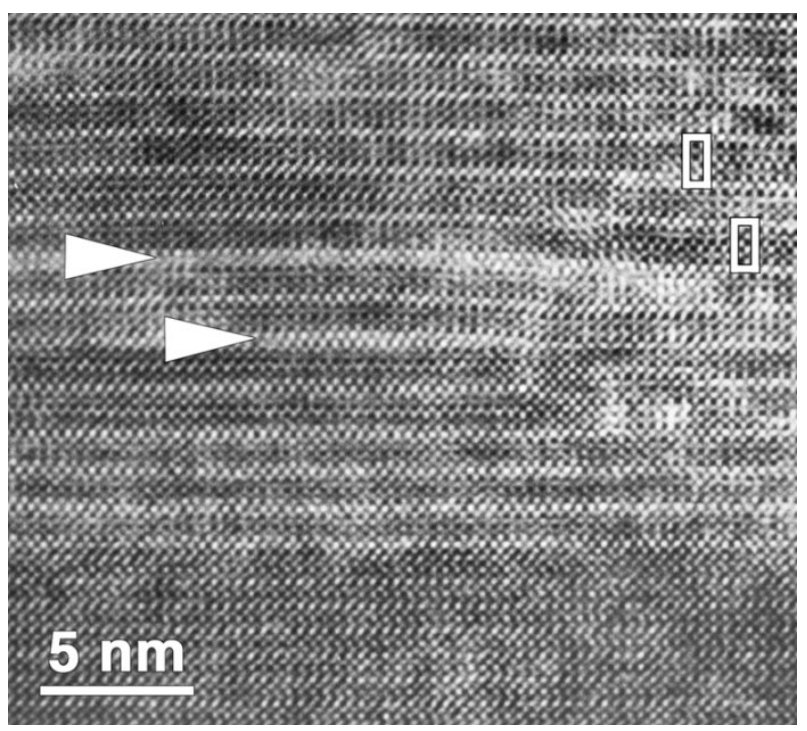

Fig. 3. Y123 thin film grown on NGO by sub-unit cell layer epitaxy. Extra blocks are marked by arrowheads, whereas white rectangles indicate Y124 defects.

therefore be manipulated by depositing a single unit cell buffer layer from a target with cation ratio $\mathrm{Y}: \mathrm{Ba}: \mathrm{Cu}$ equal to $1: 2: 2$ before subsequent deposition of Y123. HREM cross-section images (Fig. 2) prove that this results in high quality films with good epitaxial properties $\left([001]_{Y 123} / /[001]_{\mathrm{STO}}\right.$ and $[100]_{Y 123}$ $\left./ /[100]_{\mathrm{STO}}\right)$. The misfit between the film and the substrate (about $2 \%$ ), causes local bending of the film but interface dislocations are not observed.

APB's occur only occasionally. In general, APB's can be formed at unit cell steps at the surface of the substrate, present because of the miscut [8]. The spacing between the APB's in this film is comparable with the terrace width of the substrate and therefore, it can be concluded that APB's are only formed at unit cell steps at the surface of the substrate and not as a result of a variable interface configuration. Consequently, the interface configuration in this film is of type A and the formation of domains with a type B interface is suppressed.

2) Sub-Unit Cell Layer Epitaxy: The epitaxy is perfect all over the film with $[001]_{Y 123} / /[001]_{\text {NGO }}$ and $[100]_{Y 123}$ $/ /[1 \overline{1} 0]_{\mathrm{NGO}}$. Fig. 3 shows a film with a very good quality, meaning that the correct number of pulses for the individual blocks was used during deposition. However, structural defects can sometimes be observed. In Fig. 3, extra $\mathrm{BaCuOx}$ and/or $\mathrm{YCuOx}$ blocks inserted in the matrix are marked by arrowheads, whereas white rectangles indicate local $\mathrm{YBa}_{2} \mathrm{Cu}_{4} \mathrm{O}_{x}$ (Y124) defects, which have an extra $\mathrm{Cu}-\mathrm{O}$ plane inserted. The 


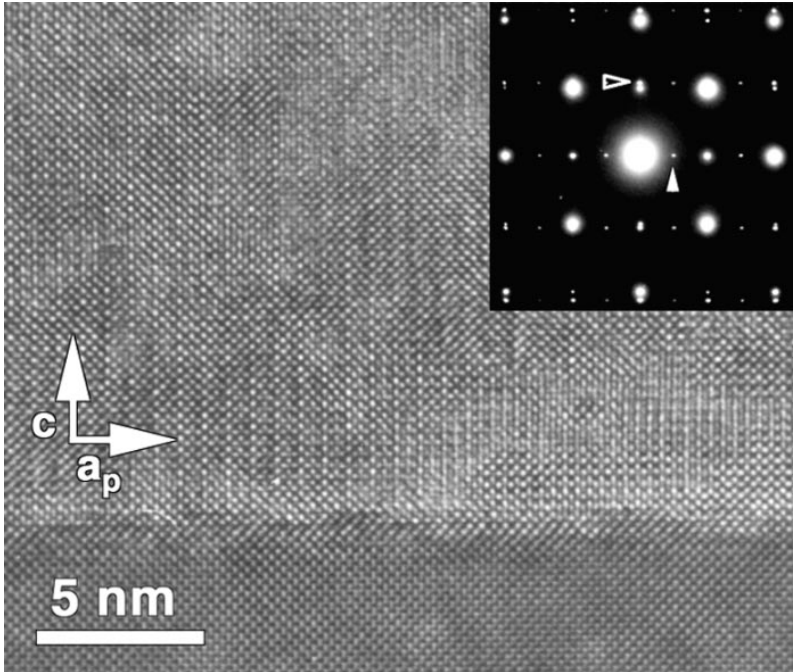

Fig. 4. HREM cross-section image of the IL thin film showing the presence of a modulation (which appears here as a doubling of the periodicity along $a_{p}$ ). In the diffraction pattern (inset), an open arrowhead indicates spots corresponding to the $c$-axis of the IL and a filled arrowhead marks spots that correspond to the modulation.

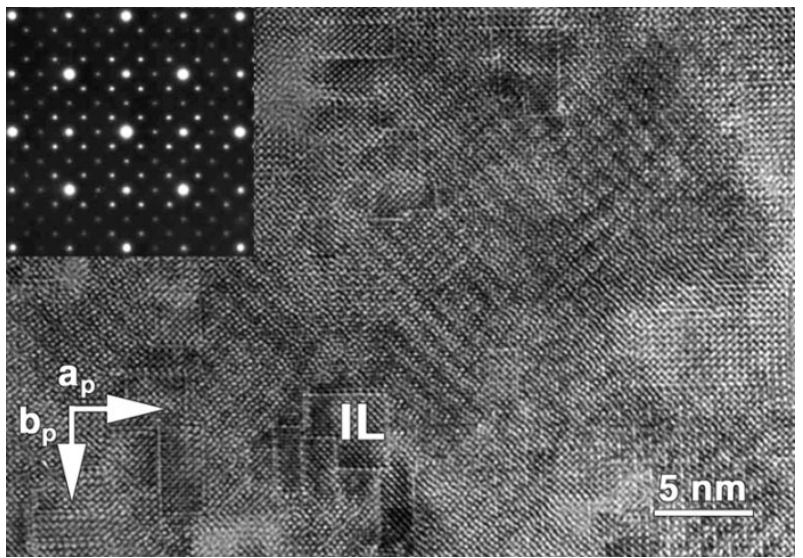

Fig. 5. Planview HREM image of the IL layer thin film. Modulation free domains (one is marked "IL) are surrounded by double $\mathrm{CuO}_{2}$ layers. Heavy spots in the diffraction image correspond to the perovskite structure, whereas weaker spots correspond to the modulated structure.

structural defects are introduced during growth and are apparently not healed by the annealing procedure after deposition.

The fact that APB's are only occasionally observed, suggests a single type of interface configuration. During growth, it was found that two unit cell thick $\mathrm{BaCuO}_{x}$ layers are sufficiently stabilized by the STO substrate, whereas $\mathrm{YCuO}_{x}$ is only stabilized in the Y123 structure [1]. Therefore, the first 2 blocks of the film are $\mathrm{BaCuO}_{x}$ blocks and consequently the interface configuration in these films must be of type B.

\section{B. $\mathrm{Sr}_{0.85} \mathrm{La}_{0.15} \mathrm{CuO} \mathrm{O}_{2}$ IL Thin Films}

1) $\mathrm{Sr}_{0.85} \mathrm{La}_{0.15} \mathrm{CuO} \mathrm{O}_{2}$ IL Thin Films on STO: None of the IL thin films on STO presented in this study are superconducting. In the films, annealed in $\mathrm{O}_{2} / \mathrm{N}_{2}$, a modulated structure is observed, which can be characterized as a $2 \sqrt{ } 2 a \times 2 \sqrt{ } 2 b \times c$ modulation combining cross-section (where the modulation can be seen as a doubling of the periodicity along $a_{p}$; Fig. 4) and planview images (Fig. 5). The $c$-axis lattice parameter of the

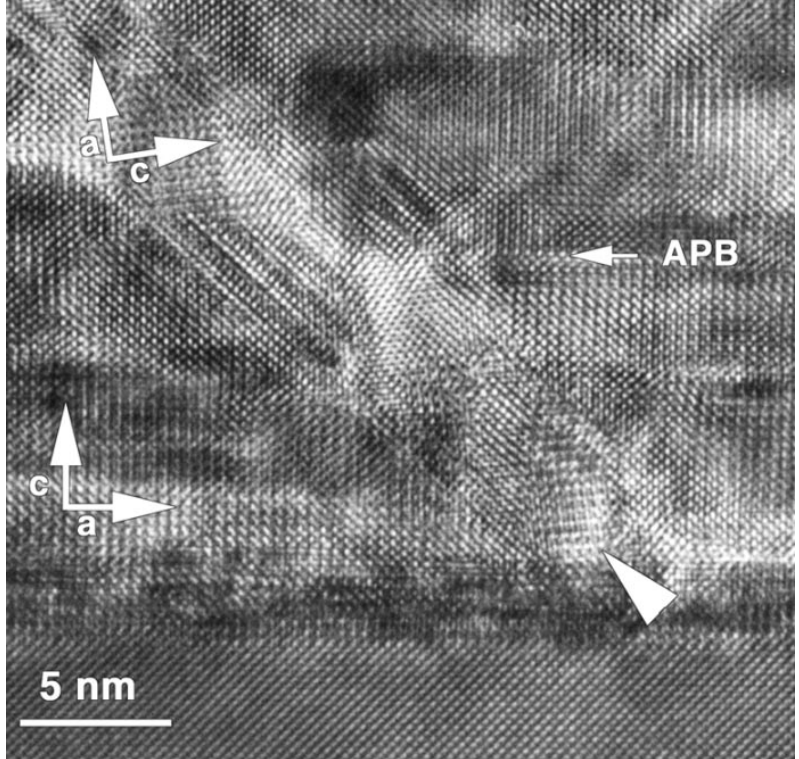

Fig. 6. HREM cross-section image of a $\mathrm{Sr}_{0.85} \mathrm{La}_{0.15} \mathrm{CuO}_{2}$ film on STO. In this film, APB's and tilted domains (marked by an arrowhead) are present.

films $(0.37 \mathrm{~nm})$, found by XRD and TEM diffraction patterns, is rather high compared to the bulk value for $\mathrm{SrCuO}_{2}(0.34 \mathrm{~nm})$.

The model we propose for the modulated structure is the same as the one proposed by Mercey et al. to explain a similar modulation in $\mathrm{Sr}_{0.75} \mathrm{Sm}_{0.25} \mathrm{CuO}_{2}$ thin films [9]. This model consists of an ordering of the $\mathrm{Sr}$ and $\mathrm{Sm}$ (in our case La) cations and implies an incorporation of oxygen at the apex positions, leading to a higher $c$-axis of the film. The discrepancy between the composition of the IL in the model $(S r / \mathrm{La}=3)$ and the nominal composition of the $\mathrm{Sr}_{0.85} \mathrm{La}_{0.15} \mathrm{CuO}_{2}$ film can be explained by taking into account the modulation-free domains both found in cross-section (not shown in Fig. 4) and planview images. Modulation free domains (one of them is marked "IL" in Fig. 5) are surrounded by loops with their sides along to the perovskite directions. Image simulations allow to characterize these loops as double $\mathrm{CuO}_{2}$ layers [10].

The $2 \sqrt{ } 2 a \times 2 \sqrt{ } 2 b \times c$ modulation does not occur in films cooled down in $\mathrm{N}_{2}$. Such films show a microstructure with a high density of APB's with Burgers vector $\mathbf{R}=[001 / 2]$ or $\mathbf{R}=[01 / 20]$. Furthermore, domains (marked by an arrowhead in Fig. 6) occur in which $[001]_{\mathrm{IL}}$ is tilted about $8^{\circ}$ with respect to $[100]_{\mathrm{STO}}$, whereas in the main part of the film $[001]_{\mathrm{IL}}$ $/ /[001]_{\text {STO }}$. The misfit ${ }^{1}$ along the [100] direction in a film in which $[001]_{\mathrm{Sr}_{0.85} \mathrm{La}_{0.15} \mathrm{CuO}_{2}} / /[001]_{\mathrm{STO}}$ is $\varepsilon=-1.20 \%$ (compressive), whereas the microstructure with the tilted domains has a misfit of $\varepsilon=0.28 \%$ (tensile). This indicates that in the twinned microstructure, stress, caused by the misfit between film and substrate is released. Since the in-plane lattice parameters of $\mathrm{Sr}_{1-x} \mathrm{La}_{x} \mathrm{CuO}_{2}$ will increase by doping the structure with La [11], compressive strain, due to the misfit with STO

${ }^{1}$ The misfit was calculated using the formula:

$$
\frac{a_{\mathrm{STO}}-a_{\mathrm{IL}}}{a_{\mathrm{IL}}}=\varepsilon
$$

For the sample with the twinned domains, the value for $a_{\mathrm{IL}}$ was calculated by taking into account the average width of a tilted domain $(5.43 \mathrm{~nm})$ and the spacing between these domains $(43.75 \mathrm{~nm})$. 


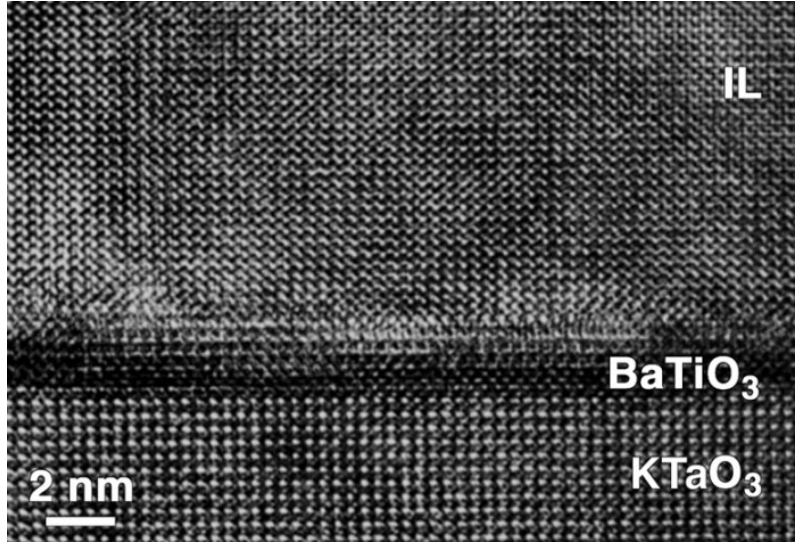

Fig. 7. $\mathrm{Sr}_{0.85} \mathrm{La}_{0.15} \mathrm{CuO}_{2}$ film on $\mathrm{KTO}$ with $\mathrm{BTO}$ buffer layer. No secondary phases are observed.

will increase with increasing La doping. For a certain doping, stress will be released by the formation of a modulated structure or by the formation of a twinned microstructure. However, the presence of a $2 \sqrt{ } 2 a \times 2 \sqrt{ } 2 b \times c$ structure with oxygen at the apex positions, or a high density of twin boundaries is at the same time responsible for the absence of superconductivity in these films.

2) $\mathrm{Sr}_{0.85} \mathrm{La}_{0.15} \mathrm{CuO}_{2}$ IL Thin Films on KTO: Obviously the misfit between film and substrate, should be reduced, which can be done using a KTO substrate (lattice parameters: $a=b=c=$ $0.399 \mathrm{~nm}$ ). Recently $\mathrm{Sr}_{0.90} \mathrm{La}_{0.10} \mathrm{CuO}_{2}$ thin films on KTO with $T_{c}=41.5 \mathrm{~K}$ were grown by molecular beam epitaxy [12]. In this study (using PLD), $\mathrm{Sr}_{0.85} \mathrm{La}_{0.15} \mathrm{CuO}_{2}$ thin films with $T_{c}$ of $16 \mathrm{~K}$ were obtained. This might be surprising since optimal $T_{c}$ is expected for La doping of 0.15 [13]. The reason for the lower value of $T_{c}$ can be found in the fact that $\mathrm{Sr}_{0.85} \mathrm{La}_{0.15} \mathrm{CuO}_{2}$ films on KTO are not single-phase. Whereas the main part of the film consists of the pure IL phase, secondary phases such as the modulated structure, $\mathrm{Sr}_{14} \mathrm{Cu}_{24} \mathrm{O}_{x}$ and $\mathrm{Sr}_{2-z} \mathrm{La}_{Z} \mathrm{CuO}_{x}$ are present in the film. Furthermore, expansive stress due to the misfit between KTO and the film is now partially released by the formation of interface dislocations. Single-phase $\mathrm{Sr}_{0.85} \mathrm{La}_{0.15} \mathrm{CuO}_{2}$ films with a perfect IL structure can be obtained, using a BTO buffer layer with lattice parameters $a=b=c=0.403 \mathrm{~nm}$ (Fig. 7). However, these films have a lower $T_{c}(12.5 \mathrm{~K})$ compared to those directly deposited on KTO. It seems that tensile strain, which is even higher than in the films on KTO without a BTO buffer layer lowers $T_{c}$.

\section{CONCLUSIONS}

HREM proves that high quality Y123 thin films with a single type of interface configuration can be obtained by sub-unit cell layer epitaxy or by using a single unit cell layer of Y122.

In $\mathrm{Sr}_{0.85} \mathrm{La}_{0.15} \mathrm{CuO}_{2}$ IL thin films, grown on STO, compressive stress is released by the formation of a modulated or twinned structure. Both mechanisms however eliminate superconductivity. To lower the misfit between the film and the substrate, a KTO substrate was used, resulting in superconducting films, which are only single-phase when a BTO buffer layer is used.

\section{REFERENCES}

[1] G. J. H. M. Rijnders, "The Initial Growth of Complex Oxides: Study and Manipulation," Ph.D., Univ. of Twente, Dept. of Appl. Phys, Enschede, The Netherlands, 2001.

[2] G. J. H. M. Rijnders, G. Koster, D. H. A. Blank, and H. Rogalla, Appl. Phys. Lett, vol. 70, pp. 1888-1890, 1997.

[3] S. Bals, R. Rijnders, D. H. A. Blank, and G. Van Tendeloo, Physica C, vol. 355, pp. 225-230, 2001.

[4] J.-P. Locquet, J. Perret, J. Fompeyrine, E. Mächler, J. W. Seo, and G. Van Tendeloo, Nature, vol. 394, pp. 453-456, 1998.

[5] G. Koster, G. J. H. M. Rijnders, D. H. A. Blank, and H. Rogalla, Appl. Phys. Lett, vol. 74, pp. 2920-2922, 1999.

[6] - Appl. Phys. Lett, vol. 73, pp. 2920-2922, 1998.

[7] S. Bals, G. Van Tendeloo, M. Salluzzo, and I. Maggio-Aprile, Appl. Phys. Lett, vol. 79, pp. 3660-3662, 2001.

[8] J. G. Wen, C. Traeholt, and H. W. Zandbergen, Physica C, vol. 205, pp. $354-362,1993$.

[9] B. Mercey, A. Gupta, M. Hervieu, and B. Raveau, Jour. Solid. State Chem, vol. 116, pp. 300-306, 1995.

[10] - Jour. Solid. State. Chem, vol. 116, pp. 37-44, 1995.

[11] G. Er., Y. Miyamoto, F. Kanamaru, and S. Kikkawa, Physica C, vol. 181, pp. 206-208, 1991.

[12] S. Karimoto, K. Ueda, M. Naito, and T. Imai, Appl. Phys. Lett, vol. 79, pp. 2767-2769, 2001.

[13] H. Takagi, S. Uchida, and Y. Tokura, Phys. Rev. Lett, vol. 62, pp. 1197-1200, 1989. 